\title{
Motor Planning as an Optimization of Command Representation
}

\author{
Shiro Ikeda \\ and \\ Yutaka Sakaguchi
}

The Institute of

Statistical Mathematics

4-6-7 Minami-Azabu, Minato-ku, Tokyo, 106-8569, Japan 


\title{
Motor Planning as an Optimization of Command Representation
}

\author{
Shiro Ikeda \\ The Institute of Statistical Mathematics \\ Minato-ku, Tokyo, 106-8569, Japan \\ shiro@ism.ac.jp \\ Yutaka Sakaguchi \\ The University of Electro-Communications \\ Chofu-shi, Tokyo, 182-8585, Japan \\ sakaguchi@is.uec.ac.jp
}

12 September 2007

\begin{abstract}
The fundamental problem of the motor neuroscience is to understand how humans make precise movements effortlessly. The problem seems difficult since there are infinite possible trajectories and the muscles are generally redundant. We discuss the problem from the viewpoint of motor command representation and show that a simple strategy can solve the problem with a two joints arm model. We also discuss the emergence of the muscle synergies, which may enable us to make natural motor behaviors with small degrees of freedom.
\end{abstract}

\section{Introduction}

When a target is given, humans can easily control an arm to reach it. Although there are infinite candidates of trajectories and velocity profiles to achieve the task, typical trajectories are gently curved and velocity profiles are bell-shaped. Also, our muscles to drive the arm are redundant. One of the fundamental problems of the motor neuroscience is to understand how human creates a set of appropriate motor commands to achieve the task.

In 1980's, it was shown that the criteria based on physical quantities, such as the minimum jerk (Flash \& Hogan, 1985) and the minimum changes in joint torques (Uno et al., 1989), describe the trajectory and velocity profile well. In 1998, Harris and Wolpert reported that the assumption of signal dependent noise describes many characteristics of the motor control (Harris \& Wolpert, 
1998). Recently, Haruno and Wolpert (2005) showed that the signal dependent noise gives a clue to solve the muscle redundancy.

In this paper, we solve the problem from a different viewpoint. Every trajectory and velocity profile with redundant muscles' activations has a corresponding motor command. Therefore, choosing a single motor command, which we call motor planning, is equivalent to choosing one motor command. We do not consider any physical quantities, such as jerk or minimum torque change, nor noise, but assume the functional form of motor commands with some parameters. We call the parameters the "motor command representation," and define a cost function which describes a preference of the representation. We propose a strategy to choose a single motor command based on the preference. The proposed strategy had been checked with a single joint arm (Sakaguchi \& Ikeda, 2007). In this report it is further checked with reaching tasks of a two joints arm. We show the resulting motor commands possess typical characteristics of the human reaching.

The muscle synergies are defined as the coherent activations of a group of muscles (d'Avella et al., 2003). We show that the motor commands obtained through our strategy form groups of motor commands which are similar to muscle synergies.

\section{Optimizing motor command representation}

Every motor command is a time series sent from brain to muscles. Here, we pose a question, "what kind of time series can brain create and send?" The answer to this question has not been made clear in the motor neuroscience.

In this paper, we assume the functional representation of the motor command as a linear combination of a prefixed basis, more precisely as follows

$$
u_{i}(t)=\sum_{j} w_{i j} \phi_{j}(t), \quad w_{i j} \geq 0
$$

where $u_{i}$ is the motor command to muscle $i$ and $\left\{\phi_{j}\right\}$ is the basis. Since $W=\left(w_{i j}\right)$ defines the motor command, we call it a "motor command representation." The basis is defined as a set of synchronizing patterns with different durations, which is similar to what is discussed in Houk et al. (1996).

Now, our problem is to select a single motor command representation $W$. We solve this problem by assuming sparsity. Olshausen and Field (1996) assumed sparsity on the visual representation and discussed the optimal basis, which has the characteristics of the simple cells. Here, we assume the basis and compute the optimal representation based on sparsity. We define the following cost function

$$
\text { Preference }\left(W ; \lambda_{1}, \lambda_{2}\right)=\lambda_{1} \sum_{i j} w_{i j}+\lambda_{2} \sum_{i j} w_{i j}^{2}, \quad \lambda_{1}, \lambda_{2}>0 .
$$

We would like to minimize this function which is our "preferred representation." Note that the first term is important for the sparsity. Similar idea was proposed in Zou and Hastie (2005). 
We give a simple example. Suppose $u$ is the input of the following linear forward dynamics of $x$,

$$
\dot{x}=f x+g u(t)=f x+g \sum_{j} w_{j} \phi_{j}(t),
$$

Assuming $\Phi_{j}(t)$ is the response of the system when $u=\phi_{j}(t)$, then from the linearity of the system,

$$
x(t)=\sum_{j} w_{j} \Phi_{j}(t) .
$$

When the achievement of a task is evaluated with a function Error $(x(t))$ of $x(t)$, such as the endpoint error, our proposal is to select the $\left\{w_{j}\right\}$ which minimizes

$$
\text { Cost }=\operatorname{Error}(x(t))+\operatorname{Preference}\left(W ; \lambda_{1}, \lambda_{2}\right)=\operatorname{Error}\left(W:\left\{\Phi_{j}(t)\right\}\right)+\operatorname{Preference}\left(W ; \lambda_{1}, \lambda_{2}\right) .
$$

If $\operatorname{Error}(x(t))$ is a linear or quadratic function of $x(t)$, Cost becomes a quadratic function of $\left\{w_{j}\right\}$, and optimal $W$ is solved with a quadratic programming (QP) method.

In the following, we use a two joints arm, which is a nonlinear system, to show how the proposed strategy works.

\section{Model}

\subsection{Two joints arm}

In this paper, we consider a 2-joints (shoulder and elbow) 6-muscles arm. This model has been widely studied (Katayama \& Kawato, 1993; Li \& Todorov, 2004; Oyama \& Uno, 2006). We first describe the dynamics and the parameters which are used in our experiments.

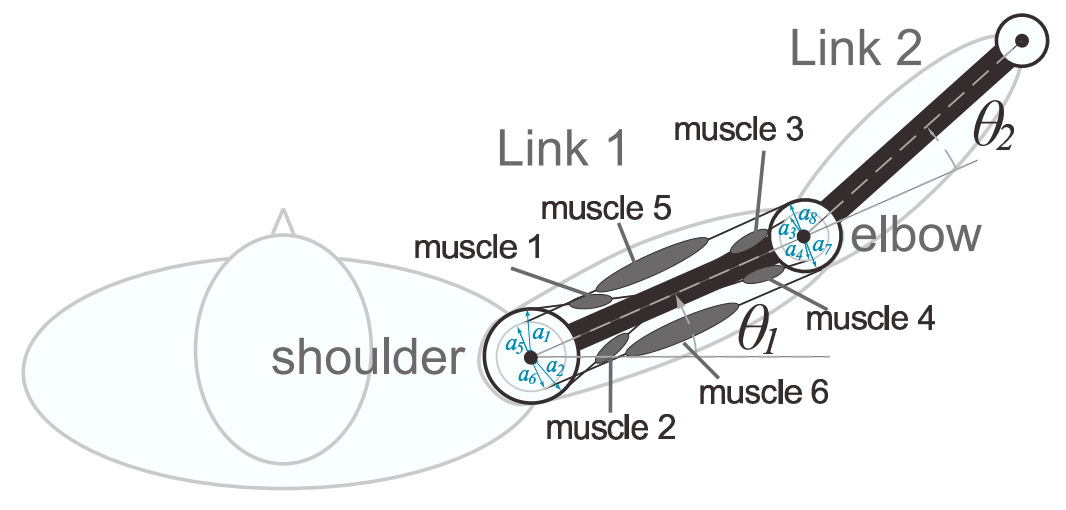

Figure 1: Two joints six muscles arm.

The model is shown in figure 1. The inverse dynamics of the arm in the horizontal plane is

$$
M(\boldsymbol{\theta}) \ddot{\boldsymbol{\theta}}+C(\boldsymbol{\theta}, \dot{\boldsymbol{\theta}})+B \boldsymbol{\theta}=\boldsymbol{\tau}(t) .
$$


The forward dynamics becomes

$$
\ddot{\boldsymbol{\theta}}=-M(\boldsymbol{\theta})^{-1}(C(\boldsymbol{\theta}, \dot{\boldsymbol{\theta}})+B \boldsymbol{\theta}-\boldsymbol{\tau}(t)),
$$

where $\boldsymbol{\theta}(t)=\left(\theta_{1}(t), \theta_{2}(t)\right)^{T} \in \Re^{2}$ is the angle vector ( $\theta_{1}$ : shoulder, $\theta_{2}$ : elbow), and $\boldsymbol{\tau}(t)=$ $\left(\tau_{1}(t), \tau_{2}(t)\right)^{T} \in \Re^{2}$ is the torque vector, $\left(\tau_{1}\right.$ : shoulder, $\tau_{2}$ : elbow). The parameters are given as

$$
\begin{gathered}
M(\boldsymbol{\theta})=\left(\begin{array}{cc}
\alpha_{1}+2 \alpha_{2} \cos \theta_{2} & \alpha_{3}+\alpha_{2} \cos \theta_{2} \\
\alpha_{3}+\alpha_{2} \cos \theta_{2} & \alpha_{3}
\end{array}\right) \\
C(\boldsymbol{\theta}, \dot{\boldsymbol{\theta}})=\left(\begin{array}{c}
-\dot{\theta}_{2}\left(2 \dot{\theta}_{1}+\dot{\theta}\right) \\
\alpha_{3}+\alpha_{2} \cos \theta_{2}
\end{array}\right) \alpha_{2} \sin \theta_{2}, \quad B=\left(\begin{array}{cc}
\beta_{11} & \beta_{12} \\
\beta_{21} & \beta_{22}
\end{array}\right) . \\
\alpha_{1}=I_{1}+I_{2}+m_{2} l_{1}^{2}, \quad \alpha_{2}=m_{2} l_{1} s_{2}, \quad \alpha_{3}=I_{2},
\end{gathered}
$$

where, $I_{i}$ is the moment of inertia of each link $\left(I_{1}=0.029 \mathrm{kgm}^{2}, I_{2}=0.042 \mathrm{kgm}^{2}\right), m_{2}$ is the weight of link $2(1.077 \mathrm{~kg}), l_{i}$ is the length of link $i\left(l_{1}=0.275 \mathrm{~m}, l_{2}=0.345 \mathrm{~m}\right), s_{2}$ is the distance from the joint center to the center of the mass of link $2(0.162 \mathrm{~cm})$, and $\beta_{11}=1.445, \beta_{12}=\beta_{21}=0.301$, $\beta_{22}=1.383$. The parameters are the same as those in Oyama and Uno (2006).

In our experiment, when the torque is given, the forward dynamics is computed with the modified Euler method, where the updating time is 5 msec.

\subsection{Torque and moment arm}

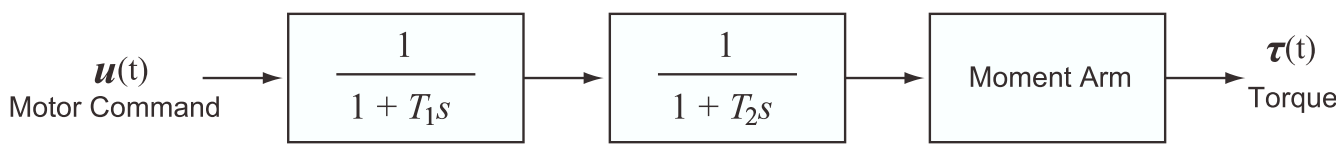

Figure 2: The process from motor command to torque.

A set of motor commands, $\boldsymbol{u}(t)=\left(u_{1}(t), \cdots, u_{6}(t)\right)^{T},\left(u_{i}(t) \geq 0\right)$ activates 6 muscles and tensions of them are combined to give the two dimensional torque $\boldsymbol{\tau}(t)$. Figure 2 shows the model of the process. We assumed $\boldsymbol{u}(t)$ is processed through 2 low-pass filters (1st-order low-pass filters with, $T_{1}=30 \mathrm{msec}, T_{2}=40 \mathrm{msec}$ ). We assume the tension of each muscle is proportional to the output of low-pass filters, which we define $\boldsymbol{u}^{\prime}(t)$. Since each muscle has a different strength depending on the cross-sectional area, unit-less $\boldsymbol{u}^{\prime}(t)$ is correctly scaled to give the tension $\boldsymbol{T}(t)$ as

$$
\boldsymbol{T}(t)=D \boldsymbol{u}^{\prime}(t),
$$

where $D$ is a diagonal matrix with $\operatorname{diag}\left(d_{1}, \cdots, d_{6}\right)=(840 \mathrm{~N}, 800 \mathrm{~N}, 560 \mathrm{~N}, 480 \mathrm{~N}, 200 \mathrm{~N}, 240 \mathrm{~N})$. The diagonal elements correspond to the maximum tension of the muscles, computed by the crosssectional area of each muscle (Wood et al., 1989) and the maximum tension per unit area (we set 
it to $62 \mathrm{~N} / \mathrm{cm}^{2}$ ). Finally $\boldsymbol{T}(t)$ is multiplied by the moment arm (figure 1 ) to give the torque as

$$
\boldsymbol{\tau}(t)=A \boldsymbol{T}(t)=A D \boldsymbol{u}^{\prime}(t), \quad A=\left(\begin{array}{cccccc}
a_{1} & -a_{2} & 0 & 0 & a_{5} & -a_{6} \\
0 & 0 & a_{3} & -a_{4} & a_{7} & -a_{8}
\end{array}\right)
$$

In this paper, we assumed a constant moment arm $\left(a_{1}, \cdots, a_{8}\right)=(3.5 \mathrm{~cm}, 4.1 \mathrm{~cm}, 2.7 \mathrm{~cm}, 2.0 \mathrm{~cm}$, $2.9 \mathrm{~cm}, 4.3 \mathrm{~cm}, 2.5 \mathrm{~cm}, 2.3 \mathrm{~cm})$.

\subsection{Motor command}

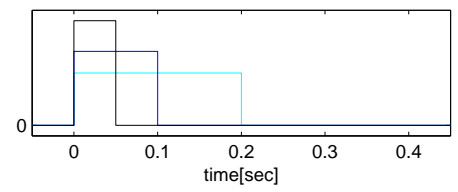

(a)

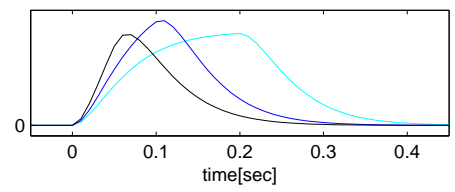

(b)

Figure 3: (a) a set of function for motor command $\phi_{i}(t)$, (b) the outputs of each function through low-pass filters $\phi_{i}^{\prime}(t)$.

As described in section 2, we assume that the motor command is represented as a linear mixture of a basis, which is a set of pre-fixed functions. In this paper, we defined a basis with 3 kinds of positive square wave functions (with length of $0.05 \mathrm{sec}, 0.1 \mathrm{sec}, 0.2 \mathrm{sec}$ ) shown in figure 3(a). Let us define them as $\phi_{i}(t), i=1,2,3$. This basis is prepared at every $T_{s}$ sec, which corresponds to a synchronous pattern generated by the brain. In out experiment, $T_{s}$ is set to 0.3 sec. Thus, a motor command $u_{i}(t)$ is written as

$$
u_{i}(t)=\sum_{k=0}^{K} \sum_{j=1}^{3} w_{i j k} \phi_{j}\left(t-k T_{s}\right), \quad w_{i j k} \geq 0
$$

The output of the low-pass filters $u_{i}^{\prime}(t)$ is written with a linear mixture of $\left\{\phi_{i}^{\prime}(t)\right\}$ which are the low-passed version of $\left\{\phi_{i}(t)\right\}$. Figure $3(\mathrm{~b})$ shows the function.

$$
u_{i}^{\prime}(t)=\sum_{k=0}^{K} \sum_{j=1}^{3} w_{i j k} \phi_{j}^{\prime}\left(t-k T_{s}\right)
$$

Note that $u_{i}(t), u_{i}^{\prime}(t) \geq 0$ from the definition.

Finally, the motor command $\boldsymbol{u}(t)$ becomes a function of $\left\{w_{i j k}\right\}$. Let us denote $\left\{w_{i j k}\right\}$ with $\boldsymbol{w}$, and motor command is $\boldsymbol{u}(t ; \boldsymbol{w})$. We also note that $\boldsymbol{\theta}(t)$ is a function of $\boldsymbol{w}$, that is, $\boldsymbol{\theta}(t ; \boldsymbol{w})$. The motor planning is to compute the $\boldsymbol{w}$ which achieves the given task of $\boldsymbol{\theta}(t ; \boldsymbol{w})$. 


\section{Optimal motor command for reaching task}

\subsection{Reaching task and cost function}

The task of reaching is to move the hand from an initial point $\boldsymbol{\theta}_{I}$ to a terminal point $\boldsymbol{\theta}_{T}$. We define the achievement of the task with the endpoint error.

$$
\operatorname{Error}(\boldsymbol{w})=\frac{1}{T} \int_{s}^{T_{e}+T_{s}}\left|\boldsymbol{\theta}(t ; \boldsymbol{w})-\boldsymbol{\theta}_{T}\right|^{2} d t .
$$

Since we are working with a discrete time, Error is redefined as

$$
\operatorname{Error}(\boldsymbol{w})=\frac{1}{T_{s}(L+1)} \sum_{l=0}^{L}\left|\boldsymbol{\theta}\left(t_{l} ; \boldsymbol{w}\right)-\boldsymbol{\theta}_{T}\right|^{2}, \quad t_{l}=T_{e}+l \frac{T_{s}}{L} .
$$

The motor command coefficient $\boldsymbol{w}$, which achieves the reaching task, can be computed by minimizing $\operatorname{Error}(\boldsymbol{w})$, however, it does not give a unique $\boldsymbol{w}$ since the muscles are redundant.

As is discussed in the section 2, we further assume a preference on the parameters $\boldsymbol{w}$, which is defined as

$$
\operatorname{Preference}\left(\boldsymbol{w} ; \lambda_{1}, \lambda_{2}\right)=\lambda_{1} \sum_{i j k} w_{i j k}+\lambda_{2} \sum_{i j k} w_{i j k}^{2} .
$$

Note that the second term shows the power of $\boldsymbol{w}$ while the first term adds the sparsity to $\boldsymbol{w}$.

Now, we define the cost function as follows,

$$
\begin{aligned}
\operatorname{Cost}\left(\boldsymbol{w} ; \lambda_{1}, \lambda_{2}\right) & =\operatorname{Error}(\boldsymbol{w})+\operatorname{Preference}\left(\boldsymbol{w} ; \lambda_{1}, \lambda_{2}\right) \\
& =\frac{1}{T_{s}(L+1)} \sum_{l=0}^{L}\left|\boldsymbol{\theta}\left(t_{l} ; \boldsymbol{w}\right)-\boldsymbol{\theta}_{T}\right|^{2}+\lambda_{1} \sum_{i j k} w_{i j k}+\lambda_{2} \sum_{i j k} w_{i j k}^{2}
\end{aligned}
$$

The optimal $\boldsymbol{w}$, which minimizes the cost function, gives the motor command for the given reaching task.

$$
\hat{\boldsymbol{w}}=\underset{\boldsymbol{w}}{\operatorname{argmin}} \operatorname{Cost}\left(\boldsymbol{w} ; \lambda_{1}, \lambda_{2}\right)
$$

\subsection{Minimizing cost function}

Since $\boldsymbol{\theta}(t ; \boldsymbol{w})$ is a nonlinear function of $\boldsymbol{w}$, it is difficult to solve eq.(3) analytically. We locally approximate $\boldsymbol{\theta}(t ; \boldsymbol{w})$ with

$$
\boldsymbol{\theta}\left(t ; \boldsymbol{w}_{(i j k)}+\Delta\right) \simeq \boldsymbol{\theta}(t ; \boldsymbol{w})+\Delta \frac{d \boldsymbol{\theta}(t ; \boldsymbol{w})}{d w_{i j k}}
$$


where $\boldsymbol{w}_{(i j k)}+\Delta$ denotes $\Delta$ is only added to $w_{i j k}$, and minimized the cost function w.r.t. $\left\{\Delta_{i j k}\right\}$.

$$
\begin{aligned}
\operatorname{minimize} \frac{1}{T_{s}(L+1)} \sum_{l=0}^{L} \mid \sum_{i j k} \Delta_{i j k} & \frac{d \boldsymbol{\theta}\left(t_{l} ; \boldsymbol{w}\right)}{d w_{i j k}}+\boldsymbol{\theta}\left(t_{l} ; \boldsymbol{w}\right)-\left.\boldsymbol{\theta}_{T}\right|^{2} \\
& +\lambda_{1} \sum_{i j k}\left(w_{i j k}+\Delta_{i j k}\right)+\lambda_{2} \sum_{i j k}\left(w_{i j k}+\Delta_{i j k}\right)^{2} .
\end{aligned}
$$

subject to $\Delta_{i j k} \geq-w_{i j k}$.

This problem is easily solved with a QP method. The derivative $d \boldsymbol{\theta}(t ; \boldsymbol{w}) / d w_{i j k}$ is approximated by adding a small positive perturbation $\delta$ to $w_{i j k}$ and computing the resulting dynamics of $\boldsymbol{\theta}$, that is

$$
\frac{d \boldsymbol{\theta}(t ; \boldsymbol{w})}{d w_{i j k}} \simeq \frac{1}{\delta}\left(\boldsymbol{\theta}\left(t ; \boldsymbol{w}_{(i j k)}+\delta\right)-\boldsymbol{\theta}(t ; \boldsymbol{w})\right) .
$$

After optimizing $\Delta_{i j k}$, every $w_{i j k}$ is renewed as $w_{i j k}+\Delta_{i j k}$, and the process is iterated until convergence. From the experiment, we see it converges surprisingly well after 3 or 4 iterations.

\section{$5 \quad$ Experiment}

\subsection{Task set}

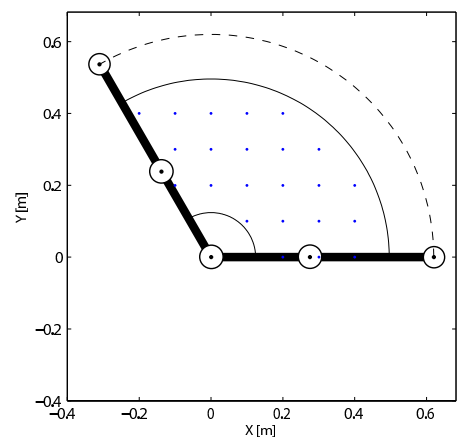

Figure 4: Candidates of the initial and terminal points.

We computed the optimal motor commands for a set of reaching tasks. Figure 4 shows the set of initial and terminal points. We first set a fan shape region, the range of $\boldsymbol{\theta}$ is $0 \leq \theta_{1} \leq 2 \pi / 3$, $\theta_{2}=0$ and distance from the origin is between $0.2 l_{a}$ to $0.8 l_{a}$, where $l_{a}=l_{1}+l_{2}$. We chose grids every $10 \mathrm{~cm}$ on horizontal plane, and if the grid falls into the fan shape region, it is a candidate of the initial and terminal points.

Every pair of points is chosen if the distance between them is more or equal to $20 \mathrm{~cm}$. There are 380 pairs which satisfies the condition, and we used all of them. This is the task set.

The values $L, T_{e}$, and $T_{s}$ in eq.(2) are set to $8,0.4 \mathrm{sec}$, and $0.4 \mathrm{sec}$, respectively, and $\lambda_{1}$ and $\lambda_{2}$ in eq.(3) are set to $2 \times 10^{-6}$ and $1 \times 10^{-5}$, respectively. 


\subsection{Results}

Out of 380 reaching tasks, 4 results are summarized in figure 5. The results in figure 5(a) show slightly curved trajectories, which are the typical characteristics of reaching tasks. The velocity profiles in figure 5(b) clearly form bell-shapes, which are also typically observed in real experiments.

Figure 5(c) shows the low-passed motor commands of 6 muscles. Many of the motor commands become 0 , that is, the motor commands are sparse, and the redundancy of muscles are clearly removed.

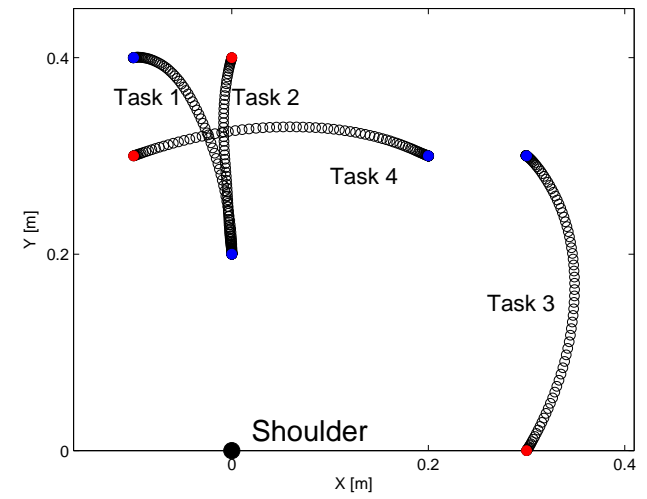

(a) trajectories
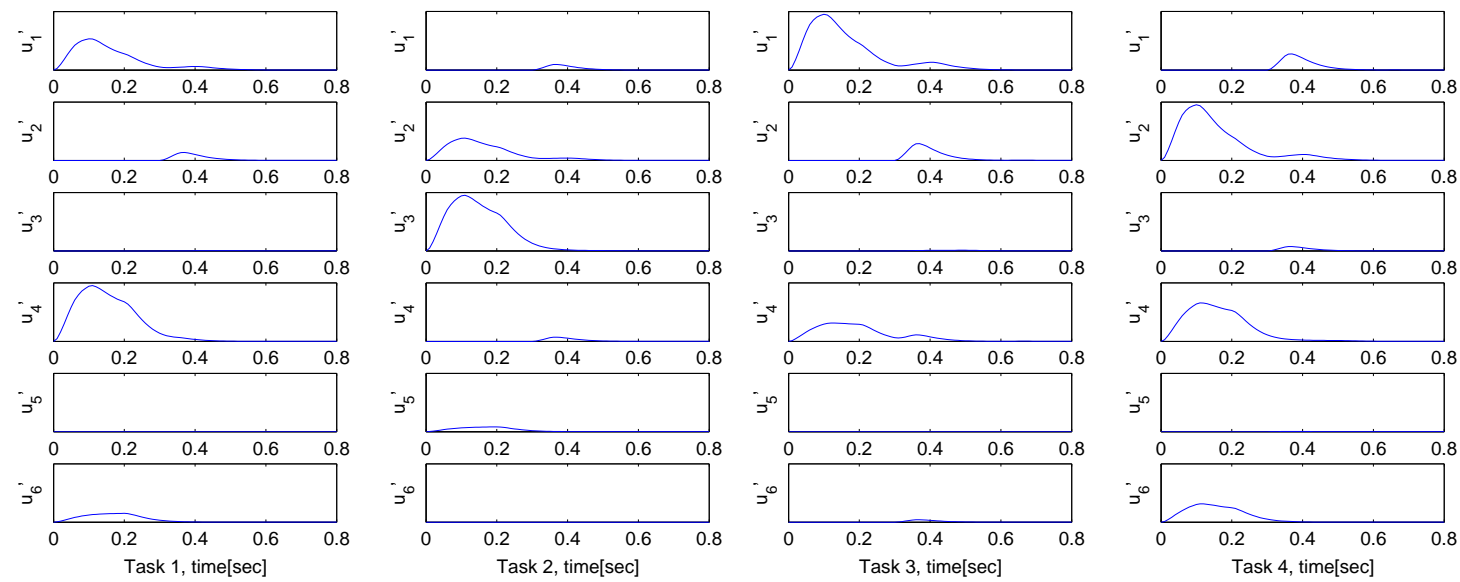

(c) low-passed motor commands of 6 muscles
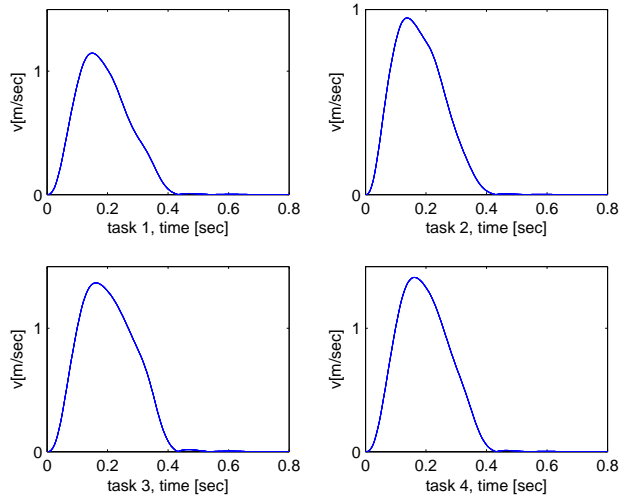

(b) velocity profiles

Figure 5: Results: 4 reaching tasks out of 380 are shown. Each task is as follows (denoted with $x-y$ coordinate $(x \mathrm{~cm}, y \mathrm{~cm}))$ : Task 1 ; from $(0,20)$ to $(-10,40)$, Task 2 ; from $(0,40)$ to $(0,20)$, Task 3 ; from $(30,0)$ to $(30,30)$, and Task 4 ; from $(-10,30)$ to $(20,30)$, (a) shows the trajectories, where red and blue dots are initial and terminal points, respectively, (b) the velocity profile of each task, (c) the low-passed motor commands of 6 muscles. 


\subsection{Synergies}

Furthermore, we observe some groups of muscles tend to be activated simultaneously. This observation motivates us to give further analysis. The idea of muscle synergies has been discussed in d'Avella et al. (2003). The muscle synergies are the "coherent activations, in space or time, of a group of muscles," which is considered to be "building blocks that could simplify the construction of motor behaviors," (from d'Avella et al. (2003)). In d'Avella et al. (2003), the NMF-type (Nonnegative Matrix Factorization) analysis is applied to the measured EMG. In our case, we apply NMF-type approach to the coefficients of the motor commands, and see if we observe some interesting results.

The motor command $\boldsymbol{u}(t)$ is represented by eq. (1). We first define the normalized coefficient vector $\boldsymbol{\omega}_{k}$ as follows,

$$
\boldsymbol{\omega}_{k}=\frac{1}{\left|\left(w_{11 k}, w_{12 k}, \cdots, w_{63 k}\right)\right|}\left(w_{11 k}, w_{12 k}, \cdots, w_{63 k}\right)^{T} .
$$

$\boldsymbol{\omega}_{k}$ is a vector of each time step and its dimension is (\# of basis functions $\times$ \# of muscles) $=$ $3 \times 6=18$. Non-zero $\boldsymbol{\omega}_{k}$ are collected from the results of 380 reaching tasks, and renumbered to form a matrix $W$ as

$$
W=\left(\boldsymbol{\omega}_{1}, \cdots, \boldsymbol{\omega}_{N}\right) .
$$

In our case, $N=1140$, which shows each of 380 tasks has 3 non-zero $\boldsymbol{\omega}_{k}$, and $W$ is a matrix with a size of $18 \times 1140$, where every component is positive and the squared length of each column is 1 .

We define synergy vectors $\boldsymbol{s}_{1}, \cdots, \boldsymbol{s}_{M}, M<18$, where $\boldsymbol{s}_{m}$ is an 18 dimensional vector with positive components. Let us define a synergy matrix $S=\left(s_{1}, \cdots, s_{M}\right)$, and assume

$$
W \simeq S H, \text { where } H=\left(h_{m n}\right) \in \Re_{+}^{M \times N} .
$$

We would like to compute $S$ and $H$ when $W$ is given. This is the NMF problem (Lee \& Seung, 1999). We solved the following problem

$$
\text { minimize }\|W-S H\|_{F}^{2}+\lambda \sum_{i m} s_{i m}, \quad \text { subject to } s_{i m} \geq 0, h_{m n} \geq 0,
$$

where $\|\cdot\|_{F}$ is the Frobenius norm and the second term makes $S$ sparse $\left(\lambda\right.$ is set to $\left.1 \times 10^{-5}\right)$. The problem is easily solved with a QP method. We applied a QP method to compute $H$ and $S$ iteratively. Although the algorithm has the initial condition dependence, it monotonically converges to a local minimum. We varied $M$ from 4 to 8 , and computed the synergies with different initial values. It is easy to imagine that as the number of the synergies increases, $\|W-S H\|_{F}^{2}$ becomes smaller, but the synergies become isolated commands on each muscle. Figure 6 shows the lowpassed outputs of synergies, when $M$ is set to 5 .

With this set of synergies $\hat{S}, W$ is reconstructed as

$$
\hat{\boldsymbol{\omega}}_{n}=\hat{S} \hat{\boldsymbol{h}}_{n}
$$

where $\hat{H}=\left(\hat{\boldsymbol{h}}_{1}, \cdots, \hat{\boldsymbol{h}}_{N}\right)$. The average squared error $\sum_{n=1}^{N}\left|\boldsymbol{\omega}_{n}-\hat{\boldsymbol{\omega}}_{n}\right|^{2} / N$ is $5.1 \%$. 


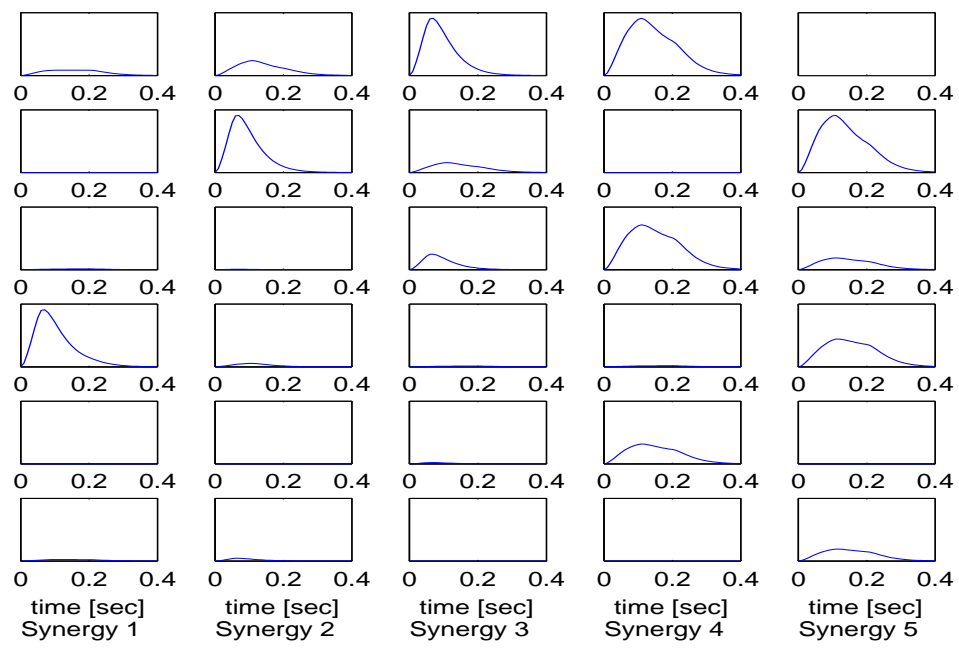

Figure 6: Synergies.

\section{Discussion and conclusion}

It has been shown that a simple assumption on the preference of the motor command representation solves the reaching task. The resulting motor commands are represented with a set of coefficients of the basis. The coefficients are sparse, and a synergy-like structure is observed.

It is difficult to imagine brain is optimizing the motor command representation for every motor planning. A more plausible idea is that brain stores learned motor commands and creates a mapping function from task to motor command, and only the mapping function is used in our daily life. In our case, the motor command is represented with a finite number of positive coefficients, and the function will have a form

$$
\boldsymbol{w}=f\left(\boldsymbol{\theta}_{I}, \boldsymbol{\theta}_{T}\right)
$$

As for the implementation, a compact representation of $\boldsymbol{w}$ is preferable. Our approach gives a sparse representation, which is suitable for the mapping function. This idea might be useful for robotics to create human-like movements.

Finally, we note that we did not assume any noise (Harris \& Wolpert, 1998) nor feedback (Li \& Todorov, 2004). It is clear that there are noises and a feedback control is necessary for precise control. Our approach can be extended naturally to implement them, and it is one of our future works. Also, biological plausible muscle models and basis functions should be considered.

\section{References}

d'Avella, A., Saltiel, P., \& Bizzi, E. (2003). Combinations of muscle synergies in the construction of a natural motor behavior. Nature Neuroscience, 6, 300-308. 
Flash, T., \& Hogan, N. (1985). The co-ordination of arm movements: An experimentally confirmed mathematical model. J. Neurosci., 5, 1688-1703.

Harris, C. M., \& Wolpert, D. M. (1998). Signal-dependent noise determines motor planning. Nature, 394, 780-784.

Haruno, M., \& Wolpert, D. M. (2005). Optimal control of redundant muscles in step-tracking wrist movements. J. Neurophysiol., 94, 4244-4255.

Houk, J. C., Buckingham, J. T., \& Barto, A. G. (1996). Models of the cerebellum and motor learning. Behavioral and Brain Science, 19, 368-383.

Katayama, M., \& Kawato, M. (1993). Virtual trajectory and stiffness ellipse during multijoint arm movement predicted by neural inverse models. Biological Cybernetics, 69, 353-362.

Lee, D. D., \& Seung, H. S. (1999). Learning the parts of objects by non-negative matrix factorization. Nature, 401, 788-791.

Li, W., \& Todorov, E. (2004). Iterative linear quadratic regulator design for nonlinear biological movement systems. Proceedings of the 1st International Conference on Informatics in Control, Automation and Robotics(ICINCO 2004) (pp. 222-229).

Olshausen, B. A., \& Field, D. J. (1996). Emergence of simple-cell receptive field properties by learning a sparse code for natural images. Nature, 607-609.

Oyama, T., \& Uno, Y. (2006). Estimation of a human planned trajectory from a measured trajectory. Systems and Computers in Japan, 37, 1-11.

Sakaguchi, Y., \& Ikeda, S. (2007). Motor planning and sparse motor command representation. Neurocomputing, 70, 1748-1752.

Uno, Y., Kawato, M., \& Suzuki, R. (1989). Formation and control of optimal trajectory in human multijoint arm movement. minimum torque-change model. Biological Cybernetics, 62, 275-288.

Wood, J., Meek, S., \& Jacobsen, S. (1989). Quantitation of human shoulder anatomy for prosthetic arm control-II. anatomy matrices. J. Biomech., 22, 309-325.

Zou, H., \& Hastie, T. (2005). Regularization and variable selection via the elastic net. J. R. Statistical Society, Series B, 67, 301-320. 